\title{
PROPIEDADES PSICOMÉTRICAS DE UNA \\ VERSIÓN BREVE DEL DRIVING ANGER \\ EXPRESSION INVENTORY EN \\ CONDUCTORES ESPAÑOLES
}

\section{PSYCHOMETRIC PROPERTIES OF A SHORT \\ VERSION OF THE DRIVING ANGER \\ EXPRESSION INVENTORY IN \\ SPANISH DRIVERS}

\author{
DAVID HERRERO-FERNÁNDEZ ${ }^{1}$, MiREIA OLIVA-MACÍAS ${ }^{1}$ Y \\ PAMELA PARADA-FERNÁNDEZ ${ }^{1}$
}

Cómo referenciar este artículo/How to reference this article:

Herrero-Fernández, D., Oliva-Macías, M. y Parada-Fernández, P. (2019). Propiedades psicométricas de una versión breve del Driving Anger Expression Inventory en conductores españoles [Psychometric Properties of a Short Version of the Driving Anger Expression Inventory (DAX) in Spanish Drivers]. Acción Psicológica, 16(1), 63-74. https://doi.org/10.5944/ap.16.1.22364

\section{Resumen}

La ira en la conducción y los comportamientos agresivos que se derivan de esta emoción pueden afectar negativamente el desempeño en la conducción. Para evaluar este tipo de comportamientos es necesario contar con instrumentos fiables y válidos. Uno de los más utilizados es el Driving Anger Expression Inventory, que en su forma completa consta de 50 ítems. Esto hace que se trate de un instrumento excesivamente extenso. El

Correspondence address [Dirección para correspondencia]: David Herrero Fernández. Universidad Europea del Atlántico C/ Isabel Torres, 21 39011, Santander, España. Email: d.herrero1984@gmail.com

ORCID: David Herrero Fernández (http://orcid.org/0000-0002-6092-8332), Mireia Oliva-Macías (http://orcid.org/00000002-6645-1328) y Pamela Parada-Fernández (http://orcid.org/0000-0003-2205-9495).

${ }^{1}$ Universidad Europea del Atlántico, España.

Recibido: 8 de marzo de 2018.

Aceptado: 23 de abril de 2018. objetivo de este estudio (de tipo transversal) fue desarrollar una versión breve del Driving Anger Expression Inventory que permitiera evaluar en poco tiempo la frecuencia de diferentes tipos de expresión de la ira al volante. Para ello se contó con una muestra de 441 conductores que rellenaron la versión completa del Driving Anger Expression Inventory, extrayéndose los ítems que mayores cargas factoriales mostraban a partir de un Análisis Factorial Confirmatorio. De esta manera se obtuvo una versión de 22 ítems que ajustaron satisfactoriamente en cinco factores: Expresión verbal de la ira $(\alpha=.84)$, Expresión física $(\alpha=.76)$, Expresión 
mediante el propio vehículo $(\alpha=.74)$, Expresión desplazada $(\alpha=.78)$ y Expresión adaptativa $(\alpha=.77)$. Se comentan las principales implicaciones de estos resultados, así como las limitaciones del estudio.

Palabras clave: Ira en conducción; Conducción agresiva; Expresión desadaptativa de la ira; Evaluación psicológica; Seguridad vial.

\begin{abstract}
Driving anger and its derived aggressive behaviors may affect driving negatively. In order to assess this kind of behaviors it is necessary to have reliable and valid psychometric instruments. One of the most one is the Driving Anger Expression Inventory, which in its long form is composed of 50 items. It makes it an excessive long questionnaire. The aim of the present research was to develop a brief version of the Driving Anger Expression Inventory to assess in few minutes the frequency of different types of anger expression behind the wheel. Therefore a sample composed of 441 drivers who completed the long version of the Driving Anger Expression Inventory. Afterwards, those items with highest factor loadings in the Confirmatory Factor Analysis were chosen to compose the new short form. Finally, a 22-item version was proposed. It fitted satisfactorily in five factors, similarly to the long form: Verbal anger expression $(\alpha=.84)$; Physical anger expression $(\alpha=.76)$; Expression through the vehicle $(\alpha=.74)$; Displaced expression $(\alpha=.78)$, and Adaptative expression $(\alpha=.77)$. Implications of the results are discussed.
\end{abstract}

Keywords: Driving anger; Aggressive driving; Desadaptative anger expression; Psychological assessment; Road safety.

\section{Introducción}

Los accidentes de tráfico suponen una de las principales causas de muerte en el mundo, con 1,35 millones de muertes al año y entre 20 y 50 millones de heridos (WHO,
2018). En el caso concreto de España, en el año 2016 se contabilizaron 102362 accidentes con víctimas, con un total de 1810 fallecidos. Estos datos suponen un incremento con respecto a 2015 de 4606 accidentes y de 121 fallecidos (DGT, 2018). Por tanto, se trata de un grave problema social que debe ser estudiado en aras a reducir estas cifras de siniestralidad.

Dentro de las causas de los accidentes, se han identificado tres categorías principales de variables: vehículo, vía y factor humano, siendo éste último el que mayor cantidad de varianza parece explicar de éstos (Evans, 1991). Dentro del factor humano, la ira y los comportamientos agresivos asociados a ella se han relacionado fuertemente con el incremento en la probabilidad de sufrir accidentes de tráfico (J. L. Deffenbacher, D. M. Deffenbacher, Lynch y Richards, 2003; J. L. Deffenbacher, Filetti, Richards, Lynch y Oetting, 2003)

La ira, como emoción básica, constituye una respuesta universal y frecuente ante situaciones frustrantes tanto en general (Spielberger, Krasner y Solomon, 1988) como en el contexto específico de la conducción (J. L. Deffenbacher, Oetting y Lynch, 1994). Tales situaciones han sido clasificadas en tres tipos de evento: comportamientos arriesgados de otros conductores como evento que mayor nivel de ira genera; actos de hostilidad directa (insultos, etc.) sufridos, y obstrucciones que ralentizan la marcha deseada por el conductor (Herrero-Fernández, 2012). La investigación precedente ha demostrado que la agresión conforma la principal manera de expresión ante la experiencia de esta emoción, relacionándose de forma directa con el comportamiento arriesgado al volante (HerreroFernández, Fonseca-Baeza y Pla-Sancho, 2013) y por ende afectando negativamente al correcto ejercicio de la conducción (Dahlen y Ragan, 2004; Sullman, 2015). Por este motivo, desde hace algunos años se han desarrollado instrumentos psicométricos que han tratado de medir este constructo, destacando sobre el resto el Driving Anger Expression Inventory (J. L. Deffenbacher, Lynch, Oetting y Swaim, 2002). Éste ha sido validado en muestras de distintos países como Francia (Delhomme y Villieux, 2010), Turquía (Esiyok, Yasak y Korkusuz, 2007), Malasia (Sullman, Stephens y Yong, 2014), Argentina (Trógolo, Flores y Medrano, 2018) o España (Herrero-Fernández, 2011), por poner sólo algunos ejemplos. Los resultados de estos 
y otros estudios muestran que la estructura factorial del DAX depende del país en el que se aplique y de la estrategia de análisis estadístico que se emplee. De esta manera, de acuerdo con una reciente revisión bibliográfica hecha al respecto (Alcázar-Olán, J. L. Deffenbacher, Reyes, Hernández y Casas, 2018), los factores más comúnmente observados en las diferentes versiones existentes son, en orden decreciente, el uso del vehículo para expresar la ira, la expresión verbal de la ira, y la expresión física de la ira.

Concretamente, la versión española consta de 50 ítems distribuidos en cinco factores, correspondiéndose con cinco modos diferentes de expresar la ira al volante. La primera es la expresión verbal de la ira, caracterizada por conductas tales como proferir gritos e insultos contra otros usuarios de la carretera, con el matiz de no ser escuchados por éstos. De todas ellas constituye probablemente la manera más leve de expresión agresiva, siendo además la más frecuente (Herrero-Fernández, 2012). En segundo lugar se encuentra la expresión física de la ira, caracterizada por conductas que oscilan entre los insultos y amenazas de las cuales el emisor hace deliberadamente que el objeto de las mismas sea consciente de ellas, hasta la agresión directa física. En tercer lugar se encuentra la expresión de la ira mediante el propio vehículo, que implica actos como conducir muy pegado al vehículo precedente, circular en paralelo de otro vehículo, etc., habiendo siempre un matiz de querer generar molestia o humillación en quien la recibe. En cuarto lugar se encuentra la expresión desplazada de la ira, caracterizada por tratarse de un estilo según el cual la agresión se dirige contra un objeto diferente a aquél que generó el estado de ira (Denson, Pedersen y Miller, 2006). Este factor únicamente ha sido incluido en la versión española del DAX, habiendo sido descartado en la versión original del instrumento debido a su baja consistencia interna. Finalmente, en quinto lugar se encuentra la expresión adaptativa de la ira, caracterizado por comportamientos que evitan confrontar directamente de manera agresiva con actitudes empáticas o al menos orientadas a calmar la emoción experimentada. De estas cinco maneras de expresión, las cuatro primeras se corresponden con la expresión desadaptativa de la ira, mientras que la quinta es la manera adaptativa o constructiva de hacerlo (J. L. Deffenbacher, et al., 2002; Herrero-Fernández, 2011).
Los estudios llevados a cabo en España con el DAX han mostrado que la expresión desadaptativa de la ira correlaciona de forma positiva y significativa con medidas de ira y agresión genéricas, mientras que la forma adaptativa de expresión lo hace negativamente (Herrero-Fernández, 2011, 2013a, 2013b). Igualmente, se han observado diferencias por edad, de manera que las personas más mayores presentan frecuencias más bajas de comportamientos agresivos desadaptativos que los más jóvenes. Sin embargo, al igual que en otras medidas de ira al volante, no se han encontrado diferencias significativas por sexo (Herrero-Fernández, 2011).

Sin embargo, como aspecto negativo del instrumento se ha propuesto su relativa extensión (50 ítems en el caso de la versión española), lo que en ocasiones puede dificultar su inclusión en protocolos más largos como se acostumbra a incluir en investigación psicológica. Es por ello que en la presente investigación se propone como objetivo la creación de una versión española breve del DAX, de manera que se facilite su uso en investigación de la ira y la agresión al volante garantizando las propiedades psicométricas exigibles de fiabilidad y validez.

\section{Método}

\section{Participantes}

La muestra estuvo compuesta por un total de 441 personas, de las cuales 131 (31.5\%) eran hombres y 302 $(68.5 \%)$ mujeres. La edad de los participantes oscilaba entre 18 y 71 años $(M=27.07, D T=10.58)$. En cuanto a experiencia como conductores (en términos de años con licencia de conducir) tenían entre 0 y 53 años $(\mathrm{M}=7.17$, DT $=9.71$ ). En cuanto a la frecuencia de conducción, oscilaba entre uno y siete días por semana $(\mathrm{M}=3.80$, $\mathrm{DT}=2.46$ ). Los criterios de inclusión para formar parte de la muestra fueron estar en disposición de, al menos, licencia de conducción B según el sistema español, y conducir al menos una vez por semana. Finalmente, los participantes fueron voluntarios y no recibieron ningún tipo de compensación por participar en el estudio. 


\section{Instrumentos de evaluación}

Driving Anger Expression Inventory (DAX). Se utilizó la versión completa del DAX en su versión española (Herrero-Fernández, 2011), que había sido adaptada a partir de la versión original (Deffenbacher et al., 2002). Estaba compuesta por 50 ítems que valoraban la frecuencia con que se llevaban a cabo diferentes formas de expresar la ira durante el ejercicio de la conducción. Dichas formas eran las siguientes: verbalmente (12 ítems, $\alpha=.90)$, físicamente (10 ítems, $\alpha=.79$ ), a través del propio vehículo (11 ítems, $\alpha=.82$ ), desplazadamente ( 3 ítems, $\alpha=.77$ ) y adaptativamente (14 ítems $\alpha=.81$ ). El formato de respuesta era una escala tipo Likert de entre 1 ("casi nunca") y 4 ("casi siempre").

Adicionalmente, en el protocolo del estudio se incluyeron los ítems específicos para recolectar los datos sociodemográficos (edad, sexo, años de experiencia como conductor, y número de días por semana en que se conducía).

\section{Procedimiento}

El cuestionario fue administrado por conveniencia a los participantes o bien online o bien mediante el método de lápiz y papel. El método de reclutamiento se basó en la técnica de "bola de nieve", en la que se distribuye indiscriminadamente el protocolo a través de redes sociales, correo electrónico, etc. Este criterio se justifica por la equivalencia de puntuaciones y de estructura factorial del DAX cuando se administra a través de estos dos formatos (Herrero-Fernández, 2015). Además, este sistema garantiza una mayor facilidad para reclutar a los participantes en el estudio. Antes de completar el protocolo los participantes recibieron un consentimiento informado que indicaba los objetivos del estudio, así como también el carácter anónimo, confidencial y voluntario de la investigación.

\section{Resultados}

A partir de los resultados obtenidos en el estudio original (Herrero-Fernández, 2011) se tomaron los ítems que mayor carga factorial presentaban en cada uno de los cinco factores. En total se seleccionaron 22 ítems, de modo que cinco pertenecían al factor relativo a la expresión verbal de la ira; cuatro pertenecían al factor relativo a la expresión física de la ira; cinco formaban parte del factor que valoraba la expresión de la ira a través del propio vehículo; tres valoraban la expresión desplazada de la ira, y cinco evaluaban la frecuencia de comportamientos adaptativos de la ira. Estos 22 ítems fueron analizados por medio de un Análisis Factorial Confirmatorio (AFC) llevado a cabo a partir de la matriz de covarianzas mediante el software Lisrel 8.80 (Jöreskog y Sörbom, 1997). Se siguió el método de estimación de parámetros de máxima verosimilitud debido a su robustez en el análisis de ítems politómicos (Rhemtulla, Brosseau-Liard y Savalei, 2012), y se tomó como referencia la estructura de la versión española de cinco factores. De cara a la valoración de los resultados del AFC se tuvo en cuenta tanto la significación de los nuevos pesos factoriales como el ajuste global del modelo. Esto último se valoró a través de cuatro índices de bondad de ajuste: en primer lugar, se tomó el cociente del valor de $\chi 2$ entre los grados de libertad del modelo, cuyo valor ha de ser inferior a cinco para poder dar por bueno el modelo (Wheaton, Muthén, Alwil y Summers, 1977). En segundo lugar, se analizó el Root Mean Square Error of Approximation (RMSEA) con su intervalo de confianza al $90 \%$, cuyo valor ha de ser inferior a .08 (Browne y Cudeck, 1993). Finalmente, se analizó el Comparative Fit Index (CFI) y el Non-Normed Fit Index (NNFI), cuyos valores han de ser superiores a .90 (Bentler y Bonnet, 1980). De esta forma, el resultado del ajuste de los 22 ítems en los cinco factores propuestos mostró un buen ajuste según los índices valorados, excepto en RMSEA que fue levemente

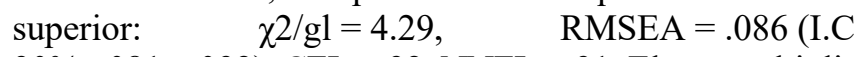
90\%: .081- .092), CFI $=.92, \mathrm{NNFI}=.91$. El test multiplicador de Lagrange mostró que el valor de $\chi 2$ se reduciría al permitir la covarianza entre un par de errores de ítems del factor 3 (ítems 11 y 13). Un nuevo análisis mostró la mejora del ajuste global del modelo en todos los índices: $\chi 2 / \mathrm{gl}=3.04, \quad \mathrm{RMSEA}=.068 \quad$ (I.C 90\%: .062-.074), $\mathrm{CFI}=.94, \mathrm{NNFI}=.94$. Posteriormente se verificó por medio del test $\chi 2$ que la mejora global del ajuste con respecto al modelo inicial era estadísticamente significativa, $\Delta \chi 2(1)=251.70, p<.001$. Por tanto, se aceptó como válido el segundo modelo, incluyendo la covarianza de un par de errores de estimación en el factor 3. El resultado de este análisis se muestra en la Tabla 1. En ella se aprecian los ítems que forman esta versión del DAX, incluyendo 
Tabla 1

Estadísticos descriptivos (Media, Desviación Típica y Asimetría), pesos factoriales y errores de estimación de los ítems y factores del DAX

\begin{tabular}{|c|c|c|c|c|c|}
\hline & M & DT & Asim. & $\begin{array}{c}\text { Peso } \\
\text { Factorial }\end{array}$ & $\begin{array}{c}\text { Error de } \\
\text { Estimación }\end{array}$ \\
\hline Expresión Verbal de la Ira $(\alpha=.84)$ & 9.63 & 3.90 & 0.94 & - & - \\
\hline 1.Insulto en voz alta a los otros conductores & 2.29 & 1.03 & 0.34 & 69 & .53 \\
\hline 2.Suelto tacos a los otros conductores en voz baja & 2.28 & 1.06 & 0.35 & .54 & .71 \\
\hline 3.Grito cosas tales como “¿dónde te habrán dado el carné?" & 1.92 & 1.04 & 0.80 & .60 & .64 \\
\hline 4.Grito a los otros conductores & 1.54 & 0.89 & 1.66 & .88 & .22 \\
\hline 5. Insulto a los otros conductores en voz alta & 1.61 & 0.94 & 1.45 & .86 & .25 \\
\hline Expresión Física de la Ira $(\alpha=.76)$ & 4.94 & 1.80 & 2.83 & - & - \\
\hline 6. Le hago un corte de mangas al otro conductor & 1.26 & 0.62 & 2.70 & 69 & .52 \\
\hline 7.Bajo la ventanilla para que se oiga mejor mi cabreo & 1.20 & 0.56 & 3.18 & .66 & .56 \\
\hline 8. Hago gestos despectivos aparte del corte de mangas & 1.31 & 0.68 & 2.41 & .75 & .44 \\
\hline 9. Trato de intimidar al otro conductor & 1.16 & 0.49 & 3.47 & .59 & .65 \\
\hline Expresión de la Ira mediante el Vehículo $(\alpha=.74)$ & 7.75 & 2.66 & 1.28 & - & - \\
\hline $\begin{array}{l}\text { 10.Sigo pegado al conductor que va delante de mí durante largo } \\
\text { tiempo }\end{array}$ & 1.34 & 0.65 & 1.99 & .56 & .69 \\
\hline 11.Conduzco un poco más rápido que antes & 2.02 & 0.92 & 0.44 & .43 & $.82^{*}$ \\
\hline 12.Acelero para molestar al otro conductor & 1.37 & 0.69 & 1.94 & .64 & .59 \\
\hline 13.Conduzco mucho más rápido que antes & 1.78 & 0.92 & 0.93 & .50 & $.75^{*}$ \\
\hline 14. Hago a los otros conductores lo que ellos me hicieron a mí & 1.24 & 0.58 & 2.69 & .66 & .56 \\
\hline Expresión Desplazada de la Ira $(\alpha=.78)$ & 4.05 & 1.60 & 1.61 & - & - \\
\hline 15.Grito a la gente que va conmigo en el vehículo & 1.36 & 0.64 & 1.71 & .55 & .70 \\
\hline 16. Expreso mi agresividad con los que van conmigo en el vehículo & 1.35 & 0.65 & 1.88 & .79 & .37 \\
\hline 17.Saco mi agresividad con otras personas al cabo de un rato & 1.34 & 0.63 & 1.89 & .89 & .21 \\
\hline Expresión Adaptativa de la Ira $(\alpha=.77)$ & 13.16 & 3.61 & 0.07 & - & - \\
\hline 18.Me digo a mí mismo que no merece la pena cabrearse & 2.69 & 0.98 & -0.02 & .72 & .49 \\
\hline 19.Intento pensar en soluciones positivas para resolver la situación & 2.60 & 0.94 & -0.02 & .66 & .56 \\
\hline $\begin{array}{l}\text { 20.Me digo a mí mismo que no merece la pena implicarse en el } \\
\text { asunto }\end{array}$ & 2.59 & 1.02 & -0.01 & .70 & .51 \\
\hline 21.Decido no rebajarme al nivel del conductor que me provoca & 2.68 & 1.14 & -0.23 & .49 & .76 \\
\hline 22. Trato de pensar en soluciones positivas para llevar a cabo & 2.59 & 0.93 & -0.04 & .62 & .61 \\
\hline
\end{tabular}

Nota. *: Los errores de estimación del par de ítems señalados correlacionaron $(\varphi=.51)$. 
los pesos factoriales, los errores de estimación, los estadísticos descriptivos y la consistencia interna de los factores.

Seguidamente, a fin de valorar la validez convergente del instrumento, se procedió a calcular la matriz de correlaciones bivariadas ( $r$ de Pearson) entre la forma larga y la forma corta del DAX. Los coeficientes de correlación entre escalas análogas de ambas versiones fueron muy altos: en el caso de la expresión verbal, $r=.91, p<.001$; en el caso de la expresión física, $r=.77, p<.001$; en el caso de la expresión mediante el propio vehículo, $r=.91$, $p<.001$; en el caso de la expresión desplazada se mantuvieron los mismos ítems; y en el caso de la expresión adaptativa de la ira, $r=.79, p<.001$.

\section{Discusión}

El objetivo de la presente investigación fue desarrollar una versión breve del DAX. Los resultados obtenidos han mostrado que la versión de 22 ítems constituye una forma fiable y válida de valorar la frecuencia con la que los conductores expresan la ira de las diferentes maneras encontradas (verbalmente, físicamente, mediante el propio vehículo, desplazadamente y adaptativamente). Por tanto, los datos obtenidos avalan su utilización en el estudio científico del comportamiento agresivo al volante.

Los resultados han mostrado un ajuste óptimo del modelo que establecía la covariación de los errores de estimación de dos ítems ("conduzco un poco más rápido que antes" y "conduzco mucho más rápido que antes"). Esto puede sugerir un cierto solapamiento entre ambos ítems, debido al contenido similar de ambos. Esto podría indicar que los conductores quizás no discriminen correctamente entre la diferencia de velocidad de conducción implicada en ambos ítems. En cualquier caso, en otras versiones de este instrumento, como la versión de Malasia (Sullman et al., 2014) o la de Turquía llevada a cabo con taxistas (Sullman, Stephens y Kuzu, 2013), se puede apreciar un elevado número de pares de errores correlacionados en los modelos confirmatorios, lo cual sugiere que posiblemente haya ítems redundantes que estén midiendo lo mismo. Por otra parte, esta nueva versión mantiene los cinco factores que fueron propuestos inicialmente por los autores de la versión original (J. L. Deffenbacher et al., 2002), inclu- yendo el que valora la expresión desplazada, que fue descartado finalmente de la versión original por la baja consistencia interna mostrada. No obstante, tal como se puede observar en los resultados de la presente investigación este factor alcanzó un nivel de consistencia interna aceptable, motivo por el cual fue introducido en el AFC. Quizás la principal desventaja de este factor radique en bajo número de ítems que lo componen. Por este motivo, de cara a futuras investigaciones se aconseja profundizar en el estudio de la agresión desplazada en la conducción, por ser sin lugar a dudas la forma de expresión agresiva menos estudiada. Como primer paso se propone la creación de nuevos ítems que puedan aportar información sobre este tipo de comportamiento, para después poder ahondar en el estudio de los correlatos psicológicos de este tipo de agresión. Teniendo en cuenta el componente principal de este tipo de agresión (expresar la ira contra un objeto distinto al que causó tal estado emocional), cabría esperar que puntuaciones altas en esta variable correlacionasen con alteraciones en otros contextos diferentes al de la conducción, tales como la vida familiar y laboral del individuo (Herrero-Fernández, 2013b; Kuppens y Mechelen, 2007).

Los resultados de esta investigación son similares a otros estudios que han obtenido versiones breves de esta herramienta con muestras de diferentes países. Por ejemplo, Stephens y Sullman (2014) desarrollaron dos versiones breves del DAX en una muestra de conductores de Gran Bretaña, una de 25 ítems y otra de 15 . En ambos casos se verificó la existencia de cuatro factores, incluyendo todos los obtenidos en la presente investigación excepto el relativo a la expresión desplazada de la ira.

En cuanto a las implicaciones prácticas de estos resultados, el hecho de haber conseguido una versión breve del DAX facilita la evaluación de la frecuencia de comportamientos agresivos en conductores en un espacio breve de tiempo, manteniendo las características exigibles de fiabilidad y validez. Esto posibilita a investigadores que quieren medir este constructo junto con otras variables, evitando la creación de protocolos de evaluación excesivamente extensos sin perder en precisión de medida.

Finalmente, este estudio presenta una serie de limitaciones. En primer lugar, el presente estudio ha evaluado la frecuencia de comportamientos agresivos al volante me- 
diante un instrumento autoinformado. Mientras que existen estudios que avalan la validez de este método (e.g., Lajunen y Summala, 2003), futuros estudios deberían verificar la validez de esta versión del DAX mediante otro tipo de medidas no autoinformadas, como por ejemplo a través de estudios de simulación o de observación a fin de verificar la validez predictiva del instrumento y de comprobar la ausencia de sesgos de respuesta. Por otro lado, sólo se ha tenido en cuenta la puntuación de los participantes en el DAX, sin aplicar otros instrumentos similares que hubiesen posibilitado la valoración de parámetros tales como la validez convergente y discriminante. Sin embargo, el hecho de haber construido esta versión breve a partir de una más extensa que sí ha verificado éstos y otros supuestos psicométricos amortigua en parte la falta de evidencia. En cualquier caso, futuros estudios deberían analizar específicamente estas características de la versión breve del DAX.

\section{Referencias}

Alcázar-Olán, R. J., Deffenbacher, J. L, Reyes, V., Hernández, L. y Casas, G. (2018). Validity of the Driving Anger Expression Inventory (DAX) in a Mexican Sample. IOSR Journal Of Humanities And Social Science, 23(7), 81-88. https://doi.org/10. 9790/0837-2307058188

Bentler, P. M. y Bonnet, D. G. (1980). Significance Test and Goodness of Fit in the Analysis of Covariance Structures. Psychological Bulletin, 88, 588-606. https://doi.org/10.1037/0033-2909.88.3.588

Browne, M. W. y Cudeck, R. (1993). Alternative Ways of Assessing Fit. In K. A. Bollen (Ed.), Testing Structural Equation Models (pp. 136-162). Newbury Park, CA: Sage:

Dahlen, E. R. y Ragan, K. M. (2004). Validation of the Propensity for Angry Driving Scale. Journal of Safety Research, 35(5), 557-563. https://doi.org/ 10.1016/j.jsr.2004.09.002
Deffenbacher, J. L., Oetting, E. R. y Lynch, R. S. (1994). Development of a Driving Anger Scale. Psychological Reports, 74(1), 83-91. https://doi.org/10.2466/pr0.1994.74.1.83

Deffenbacher, J. L., Lynch, R. S., Oetting, E. R. y Swaim, R. C. (2002). The Driving Anger Expression Inventory: A Measure of how People Express their Anger on the Road. Behaviour Research and Therapy, 40(6), 717-737. https://doi.org/10.1016/S0005-7967(01)00063-8

Deffenbacher, J. L., Deffenbacher, D. M., Lynch, R. S. y Richards, T. L. (2003). Anger, Aggression, and Risky Behavior: A Comparison of High and Low Anger Drivers. Behavior Research Therapy, 41(6), 701-718. https://doi.org/10.1016/S0005-7967(02) 00046-3

Deffenbacher, J. L., Filetti, L. B., Richards, T. L., Lynch, R. S. y Oetting, E. R. (2003). Characteristics of two Groups of Angry Drivers. Journal of Counseling Psychology, 50(2), 123-132. https://doi.org/10. 1037/0022-0167.50.2.123

Delhomme, P. y Villieux, A. (2010). Driving Anger and its Expressions: Further Evidence of Validity and Reliability for the Driving Anger Expression Inventory French Adaptation. Journal of Safety Research, 41, 417-422. https://doi.org/10.1016/ j.jsr.2010.08.003

Denson, T. F., Pedersen, W. C. y Miller, N. (2006). The Displaced Aggression Questionnaire. Journal of Personality and Social Psychology, 90(6), 10321051. https://doi.org/10.1037/0022-3514.90.6.103 2

DGT. (2018). Anuario estadístico de accidentes - 2016. [Statistical Accident Yearbook - 2016] Recuperado de http://www.dgt.es/Galerias/seguridadvial/estadisticas-eindicadores/publicaciones/anuario-estadistico-deaccidentes/Anuario-accidentes-2016.pdf. 
Esiyok, B., Yasak, Y. y Korkusuz, I. (2007). Anger Expression on the Road: Validity and Reliability of the Driving Anger Expression Inventory. Turkish Journal of Psychiatry, 18(3), 231-243.

Evans, L. (1991). Traffic Safety and the Driver. New York, NY: Van Nostrand Reinhold.

Herrero-Fernández, D. (2011). Psychometric Adaptation of the Driving Anger Expression Inventory in a Spanish sample. Differences by Age and Gender. Transportation Research Part F: Traffic Psychology and Behaviour, 14, 324-329. ttps://doi.org/10.1016/j.trf.2011.03.001

Herrero-Fernández, D. (2012). Análisis de diferencias en la magnitud de la ira provocada por distintos eventos del tráfico y en las formas de expresarla [Analysis of the Differences by Age and Gender in the Magnitude of Anger Provoked by Different Traffic Events and the Ways to Express it]. Securitas Vialis, 13, 11-23.

Herrero-Fernández, D. (2013a). Do People Change behind the Wheel? A Comparison of Anger and Aggression on and off the Road. Transportation Research Part F: Traffic Psychology and Behaviour, 21, 66-74. https://doi.org/10.1016/ j.trf.2013.09.008

Herrero-Fernández, D. (2013b). Adaptación española de la dimensión conductual del Displaced Aggression Questionnaire. Análisis de validez con medidas de ira y agresión genéricas y en la conducción [Spanish Adaptation of the Behavioural Dimension of the Displaced Aggression Questionnaire: Analysis of Validity with General and on-the-road Measures of Anger and Aggression]. Revista de Psicología Social, 28(3), 273-284. https://doi.org/ $10.1174 / 021347413807719094$

Herrero-Fernández, D. (2015). A comparison of Internet-based and Paper-and-Pencil Questionnaires in Assessing Driving Anger in a Spanish Sample. REMA. Revista Española de Metodología Aplicada, 20(1), 1-15.
Herrero-Fernández, D., Fonseca-Baeza, S. y Pla-Sancho, S. (2013). Predicción del comportamiento de riesgo al volante en base a la experiencia en conducción y a las conductas agresivas. Influencia de la edad y el género [Prediction of the Risky Behavior on the Road Based on Driving Experience and Driving Aggression. Influence of Age and Gender]. Securitas Vialis, 14, 46-57.

Jöreskog, K. y Sörbom, D. (1997). Lisrel 8: User's Reference Guide. Lincolnwood, IL: Scientific Software International.

Kuppens, P. y Mechelen, I. V. (2007). Interactional Appraisal Models for the Anger Appraisals of Threatened Self-Esteem, Other-Blame, and Frustration. Cognition and Emotion, 21(1), 56-77. https://doi.org/10.1080/02699930600562193

Lajunen, T. y Summala, H. (2003). Can we Trust Selfreports of Driving? Effects of Impression Management on Driver Behaviour Questionnaire responses. Transportation Research Part F: Traffic Psychology and Behaviour, 6(2), 97-107. https://doi.org/10.1016/S1369-8478(03)00008-1

Rhemtulla, M., Brosseau-Liard, P. E. y Savalei, V. (2012). When Can Categorical Variables be Treated as Continuous? A Comparison of Robust Continuous and Categorical SEM Estimation Methods under Suboptimal Conditions. Psychological Methods, 17(3), 354-373. https://doi.org/10.1037/a0029315

Spielberger, C. D., Krasner, S. S. y Solomon, E. P. (1988). The Experience, Expression, and Control of Anger. En M. P. Janisse (Ed.), Health Psychology: Individual Differences and Stress (pp. 89-108). New York, NY: Springer/Verlag.

Stephens, A. N. y Sullman, M. J. M. (2014). Development of a Short form of the Driving Anger Expression Inventory. Accident Analysis and Prevention, 72, 169-176. https://doi.org/10.1016/ j.aap.2014.06.021 
Sullman, M. J. M. (2015). The Expression of Anger on the Road. Safety Science, 72, 153-159. https://doi.org/10.1016/j.ssci.2014.08.013

Sullman, M. J. M., Stephens, A. N. y Kuzu, D. (2013). The Expression of Anger amongst Turkish Taxi Drivers. Accident Analysis and Prevention, 56, 4250. https://doi.org/10.1016/j.aap.2013.03.013

Sullman, M. J. M., Stephens, A. N. y Yong, M. (2014). Anger, Aggression, and Road Rage Behaviour in Malaysian Drivers. Transportation Research Part F: Traffic Psychology and Behaviour, 29, 70-82. https://doi.org/10.1016/j.trf.2015.01.006

Trógolo, M. A., Flores, P. E. y Medrano, L. A. (2018). Validez y Confiabilidad del Inventario de Expresión de Ira (DAX) en Conductores de Argentina [Validity and Reliability of the Driver Anger Expression Inventory (DAX) in Argentinean Drivers]. Revista Iberoamericana de Diagnóstico y
Evaluación - e Avaliação Psicológica, 46, 21-35. https://doi.org/ 10.21865/RIDEP46.1.02

Wheaton, B., Muthén, B., Alwil, D. y Summers, G. (1977). Assessing Reliability and Stability in Panel Models. En D. R. Heise (Ed.), Sociological Methodology (pp. 84-136). San Francisco, CA: Jossey-Bass.

WHO. (2018). Global Status Report on Road Safety 2018. Ginebra, Swizeland: Autor. Recuperado de https://www.who.int/violence_injury_prevention/ $\mathrm{r}$ oad_safety_status/2018/GSRRS2018_Summary_E N.pdf

\title{
PSYCHOMETRIC PROPERTIES OF A SHORT VERSION OF THE DRIVING ANGER EXPRESSION INVENTORY IN SPANISH DRIVERS
}

\author{
DAVID HERRERO-FERNÁNDEZ ${ }^{1}$, MiREIA OLIVA-MACÍAS ${ }^{1}$, \\ AND PAMELA PARADA-FERNÁNDEZ ${ }^{1}$
}

\section{EXTENDED SUMMARY}

\section{Introduction}

Road accidents are one of the most common cause of death around the world. In Spain, there were 102362 accidents with victims in 2016, which implies an increment with regard to 2015 of 4606 accidents (DGT,
2018). Then, it is a relevant social issue that must be studied in order to reduce this accident rate.

When analyzing the casues of road accidents, three main categories are usually identified: vehicle, infrastructure, and human factor. Human factor is the category which more ammount of variance of road accidents explains. Within human factor, anger and aggressive behavior have been strongly related to the 
likelihood of getting and accident (J. L. Deffenbacher et al., 2003).

Anger as a basic emotion is an universal response in frustratring situations both in general and in the specific context of driving (Spielberger et al., 1988). More concretely, three kind of situations have been identified: risky behaviors of other road users, direct hostility behaviors, and obstructions that minorate the speed of the vehicle (Herrero-Fernández, 2012). Previous research has shown that aggression is the main way of anger expression, and it is directly related to risky behavior. Then, aggression has a negative impact in the quality of driving. Therefore, some psychometric questionnaires have been developed with the aim of measuring anger and aggression behind the wheel. One of the most used around the world is the Driving Anger Expression Inventory (DAX), which has been used in such different countries as Turkey, France, Argentina, Malaysia, China or Spain (J. L. Deffenbacher et al., 2002). The results of this research shows that the factorial structure of the DAX depends mainly on the country and the statistic analyses that are conducted. In a recent bibliographic review it has been reported that the most commonly reported factors are the use of the own vehicle to express anger, and the verbal expression of anger.

In the specific case of the Spanish version of the DAX (Herrero-Fernández, 2011), it consists of 50 items which measure five ways of anger expression. The first way is the verbal expression of anger, which implies behaviors like calling names aloud or yielling, but those behaviors must not be detected by the target of them. This is probably the less serious kind of aggression. The second way is the physical expression of anger, which consists of such behaviors as calling names aloud when the aggressor provokes the victim being conscious about them, or getting a physical fight. The third way is the expression of anger through the own vehicle, with such behaviors as driving very close to the precedent vehicle. The fouth way of anger expression is the displaced aggression, which consists of focusing aggression against a different target from that who caused the anger state. This factor has been only included in the Spanish version of the DAX. The authors of the original version erased it due to its low reliability. Finally, the fifth way of anger expression is the adaptative way of anger expression, which such behaviors as breathing deeply, or listen to the radio in order to getting calm. Then, the first four factors are desadaptative ways of anger expression, while the fifth one is the adaptative or constructive way.

Some research conducted in Spain has shown that the desadaptative ways of anger expression correlate positively with measures of general anger and aggression, while the adaptative way of anger expression does it negatively. Samely, differences by age have been showed, so youngest drivers score higher in the desadaptative ways the the oldest. Regarding the gender, no significant differences between males and females have been found.

However, an important limitation of the DAX is its lenght (50 items), so gathering information regarding this variable can take long time. Then, the current research aims to develop a reliable and valid brief version of the Spanish version of the DAX.

\section{Method}

\section{Participants}

The sample consisted of 441 people, being 131 $(31.5 \%)$ male and $302(68.5 \%)$ female. The age of the participants ranged from 18 to $71 \quad(\mathrm{M}=27.07$, $\mathrm{SD}=10.58$ ). Driving experience (in years) ranged from 0 to $53(\mathrm{M}=7.17, \mathrm{SD}=9.71)$. Regarding the driving frequency, it ranged between 1 and 7 days per week $(\mathrm{M}=3.80, \mathrm{SD}=2.46)$. The inclussion criteria were both having al least the B-type driving lincese (according to the Spanish system), and driving at least once a week. Finally, the particpants were voluntary and they did not get any compensation because of their particpation.

\section{Instruments}

Driving Anger Expression Invcentory (DAX). The Spanish large version of the DAX was used in the current research. It consists of 50 item which assess the frequency with which drivers behave in the described way during driving. The ways of aggressive behavior are grouped into five factors: verbally ( 12 items, $\alpha=.90$ ), physically (10 
items, $\alpha=.79)$, using the vehicle (11 items, $\alpha=.82)$, displacedly ( 3 items, $\alpha=.77$ ), and adaptatively (14 items, $\alpha=.81$ ). The response system is a 4-point Likert-scale $(1=$ almost never $-4=$ almost always $)$.

Additionally, specific items to measure demographic variables (age, gender, driving frequency and experience) were included.

\section{Procedure}

The questionnaire was applied to the participants by convenience either by Internet or by paper-and-pencil, given that some research has shown that the way of gathering data with the DAX has no significant effect in the scores. Before cimpleting the questionnaire, the particpants got the informed constent explaining the main goals of the study and guarranteing the confidentiality and anonimously of the study.

\section{Results}

Based on the previous results the were got in the original Spanish study of the DAX, those items with higher factor loadings were selected. On the whole, 22 items were taken, so five of them belonged to the verbal expression factor, four of them did it to the physical expression, five of them did it to the expression through the own vehicle, three did it to the displaced aggression factor, and five of them did it to the adaptative expression factor. These 22 items were analyzed through a Confirmatory Factor Analysis (CFA), through the máximum-likelihood method of parameter estimation. The results of the CFA were assessed through both the significance of the factor loadings and the goodness of fit of the model. This last was assessed thoough four índices: the $\chi^{2} / \mathrm{df}$ rate, which should be lower than 5; the RMSEA and its $90 \%$ interval of conficence, which should be lower than .08, and the CFI and the NNFI, which should be higher than .90 .

Then, the 22 items showed a good fit to the five-factors model according to the goodness of fit indices, except for the RMSEA, which was slighly high: $\chi^{2} / \mathrm{df}=4.29$, $\mathrm{RMSEA}=.086 \quad(90 \% \quad$ I.C. $=.081-.092), \quad \mathrm{CFI}=.92$,
NNFI $=.91$. Then, the Lagrange-multiplier test showed that the $\chi^{2}$ value would decrease by letting item 11 and item 13 (which belong to the third factor) errors covariate. Then, a new analysis showed the improvement of the global fit of the model: $\chi^{2} / \mathrm{df}=3.04$, RMSEA $=.068$ $(90 \%$ I.C. $=.062-.074)$, CFI $=.94$, NNFI $=.94$. Then, this second model (with the error covariation between the pair of errors of ítem 11 and ítem 13) were accepted. The results are detailed in Table 1 of the manuscript.

Finally, with the aim of assessing the convergent validity of this new brief version of the DAX, the bivariate correlation coefficient between each pair of equivalent factors (large way and brief way) was calculated. All of them were very high: in the case of verbal expression, $r=.91, p<.001$; in the case of physical expression, $r=.77, p<.001$; in the case of using the own vehicle to express anger, $r=.91, p<.001$; and in the case of the adaptative way of expressing anger, $r=.79, p<.001$. The displaced aggression factor maintained the 3 items of the original large version.

\section{Discussion}

The aim of the current research was to develop a brief version of the DAX. The results have shown that the new 22 -item version is a reliable and valid way of assessing anger expression behind the wheel.

The results have shown an optimal fit of the model to the data in five factors, with two errors covariating (item 11: "I drive slightly quicker than I did before", and ítem 13: "I drive much quicker tan I did before"). This could imply a certain overlap between both of them, due to the similar content. From a practical viewpoint, it could indiceate that divers could not discriminate correctly between the difference in the speed implied in both items. Anyway, some versions of the DAX have been developed with a lot of pairs of errors covariated (Sullman et al., 2014). On the other hand, the current version maintains the displaced aggression factor, which was discarded in the original version of the DAX due to its low reliability. However, in the case of both the Spanish long version of the DAX and the new brief version of the DAX, this factor has attained good reliability coefficients. Perhaps the main 
weakness of this factor is the small number of ítems. Then, future research should try to develop more specific measures of displaced aggression behind the wheel. It would also let to investigate the role of this variable in driving.

The results of the current research are similar to other research that has developed brief versions of the DAX. For example, in Great Britain (Stephens y Sullman, 2014) two brief versions (25 and 15 items) were developed, verifying in both cases a four-factor structure (the same factors than in the current research except for the displaced factor).

Finally, this reseach has two main limitations. First, aggressive behaviors have been studied only though a selfreport. While there are studies suggesting the validity of this procedure, future research should confirm the results got here with other methodologies, such as simulation sudies or observation measures. Second, current reseach analyzed only the socre of the particpants in the DAX. Future research shouyld verify the validity of this new brief version of the DAX by correlating it with some other similar measures. 